\title{
Space Charge Effects and Induced Signals in Resistive Plate Chambers
}

\author{
Christian Lippmann ${ }^{a, *}$, Werner Riegler ${ }^{a}$, Bernhard Schnizer ${ }^{b}$ \\ ${ }^{\text {a } C E R N, ~ E P ~ D i v i s i o n, ~ C H-1211 ~ G e n e v a ~} 23$ \\ ${ }^{\mathrm{b}}$ Institut für Theoretische Physik, TU-Graz, Graz, Austria
}

\begin{abstract}
Using special integral representations of the solution for the static electric field of a point charge in a three layer geometry with different permittivities, we calculate the effect of the space charge on the avalanche in the gas gap of an RPC. A detailed Monte Carlo simulation was developed which allows calculation of the actual charge spectrum. Results of this simulation are presented, using the example of a triggerRPC with $2 \mathrm{~mm}$ gas gap, similar to the ones used by ATLAS [1], and a timing RPC with $300 \mu \mathrm{m}$ gas gap [2]. Finally we also present analytic solutions for the weighting field of an RPC readout strip, which allow to calculate the directly induced crosstalk and induced signals.
\end{abstract}

Key words:

RPC, Space Charge Effect, Simulation, Weighting Field PACS: 07.05.Tp, 29.40.Cs

\section{Introduction}

It is shown in another presentation during this workshop [5] that the application of standard detector physics simulations to RPCs shows good agreement with measurements for efficiency and time resolution. However, this procedure leads to average total avalanche charges that deviate from measured ones by up to seven orders of magnitude (for $300 \mu \mathrm{m}$ gap timing RPCs at $3 \mathrm{kV}$ ). Also the statistics of avalanche fluctuations lead to an exponential charge spectrum while the measured spectra show a pronounced peak. To show that this

\footnotetext{
* Corresponding author.

Email address: Christian.Lippmann@cern.ch (Christian Lippmann).

URL: http://cern.ch/lippmann (Christian Lippmann).
}

Preprint submitted to Elsevier Science 11 January 2002 
discrepancy can be explained by a huge suppression factor caused by a space charge effect, we present a detailed Monte Carlo avalanche simulation program including dynamic calculation of the electric field of the space charge. We also show simulated charge spectra and a comparison to measured data.

\section{An Avalanche Simulation Program}

The simulation of an avalanche in an RPC is done by dividing the gas gap into several steps and distributing the primary clusters onto those steps. The number of clusters per mm and the cluster size distribution are obtained using HEED [4]. Then the electrons are multiplied, using the avalanche fluctuation model also discribed in [5]. The multiplication depends on the Townsend coefficient $\alpha$ and the attachment coefficient $\eta$. The dependence of those coefficients and of the driftvelocity $v_{D}$ on the electric field is derived using MAGBOLTZ [6] and IMONTE [7]. The longitudinal and transverse diffusion coefficients $D_{L}$ and $D_{T}$ are also obtained by simulation with MAGBOLTZ.

We include the space charge effect by calculating dynamically the field of the avalanche charge in the RPC on the spot where electrons are multiplied. We use an analytic solution for the potential of a point charge in a three layer geometry with different permittivities, which has been published elsewhere [8]. This special integral representation allows easy numerical calculation. Comparison to the solution of a free charge shows a deviation of up to $80 \%$ for a $2 \mathrm{~mm}$ gap RPC. Only close to the charge the deviation is less important. Transverse diffusion is included by distributing the charge transversally onto a disk following a Gaussian distribution with a $\sigma$ increasing towards the anode ( $\sigma=D_{T} \sqrt{l}$, where $l$ is the drift distance). Integrating the solution for the point charge over the charge distribution gives the field of this disk. By summing up the fields of all the disks in the gas gap we obtain the field of a three dimensional charge distribution which is the space charge of the avalanche. A snapshot of a simulated avalanche can be seen in Fig 1. Some events show an exploding electric field. Those can be interpreted as streamers. An example can be viewed in Fig 2 .

Because of the limited conductivity of the resistive layers, electrons that reached the anode will stay there for a much longer time (milliseconds) than the signal time (nanoseconds). This is not the case for an anode made of conducting material. 


\section{Results for Timing and Trigger RPCs}

We simulated timing RPCs with a $300 \mu \mathrm{m}$ gas gap and two glass resistive plates with $2 \mathrm{~mm}$ thickness. The gas mixture consists of $\mathrm{C}_{2} F_{4} H_{2}(85 \%), i \mathrm{C}_{4} \mathrm{H}_{10}(5 \%)$ and $S F_{6}(10 \%)$. Fig 3 shows good very agreement between measured and simulated spectra. We also compared spectra for resistive and conducting anodes. The difference in the average charges is about $10 \%$. Correlations of the induced electron charge with the total signal charge are shown in Fig 4.

We also simulated trigger RPCs with a $2 \mathrm{~mm}$ gas gap and two $2 \mathrm{~mm}$ bakelite resisitive plates. The gasmixture is $C_{2} F_{4} H_{2}(97 \%), i C_{4} H_{10}(2.5 \%)$ and $S F_{6}(0.5 \%)$. A simulated spectrum for $10.2 \mathrm{kV}$ is shown in Fig 5 . The shape shows the pronounced peak also observed in experiments. At this field we have $18 \%$ streamers, which is not observed in reality. At $10.5 \mathrm{kV}$ we have $85 \%$ streamers. This fact will need some more careful investigation. Slight variations of the parameters $\alpha, \eta, D_{L}$ and $D_{T}$ can change the behaviour of the simulated avalanches significantly.

\section{The Weighting Field in an RPC}

An analytic solution for the weighting field in an RPC gas gap can be used to calculate induced signals and directly induced crosstalk. This solution has been presented and has already been published elewhere [8].

\section{Conclusion}

We simulated the detector physics of Resistive Plate Chambers, assuming only physical parameters predicted by MAGBOLTZ, HEED and IMONTE. The simulated results match the experimental ones very well. We conclude that the efficiencies and average charges of $300 \mu \mathrm{m}$ and $2 \mathrm{~mm}$ gap RPCs can be explained by standard physics and a huge suppression factor caused by a space charge effect.

\section{References}

[1] ATLAS TDR 10, CERN-LHCC-97-22

[2] P. Fonte et. al. NIM A449 (2000) 295-301 


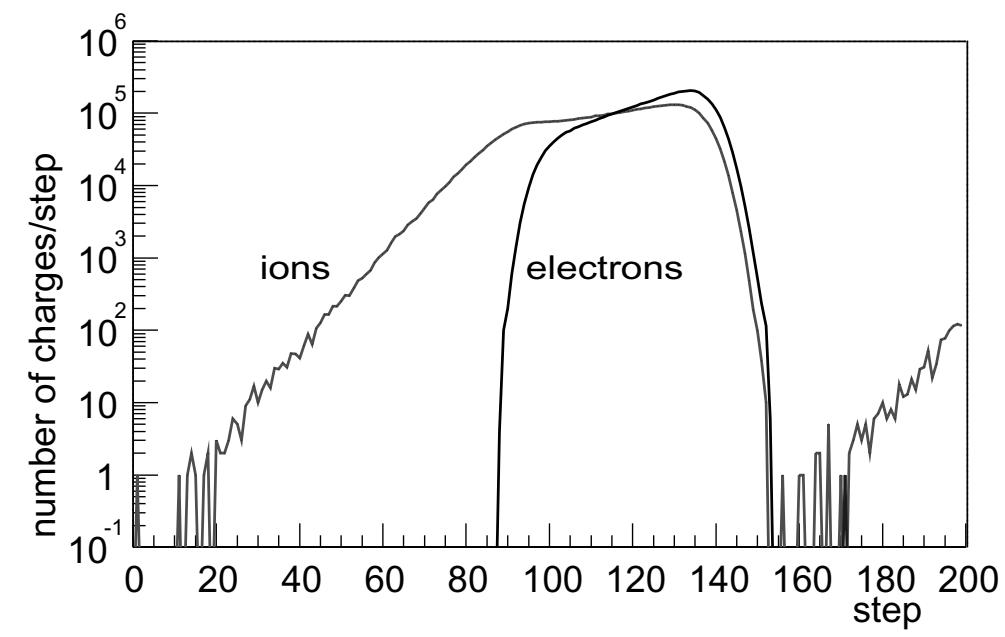

Fig. 1. Snapshot of a simulated avalanche. A $300 \mu \mathrm{m}$ gas gap is divided in 200 steps. The ion and electron distributions are shown. One cluster has already reached the anode. The space charge effect just starts to alter the Gaussian shape of the second electron cluster. At its tip and tail the electric field is about $15 \%$ higher than the applied field. Here the electrons drift faster and multipy more. At the center of the electron cloud the field is about $30 \%$ lower. In that area there is only attachment.

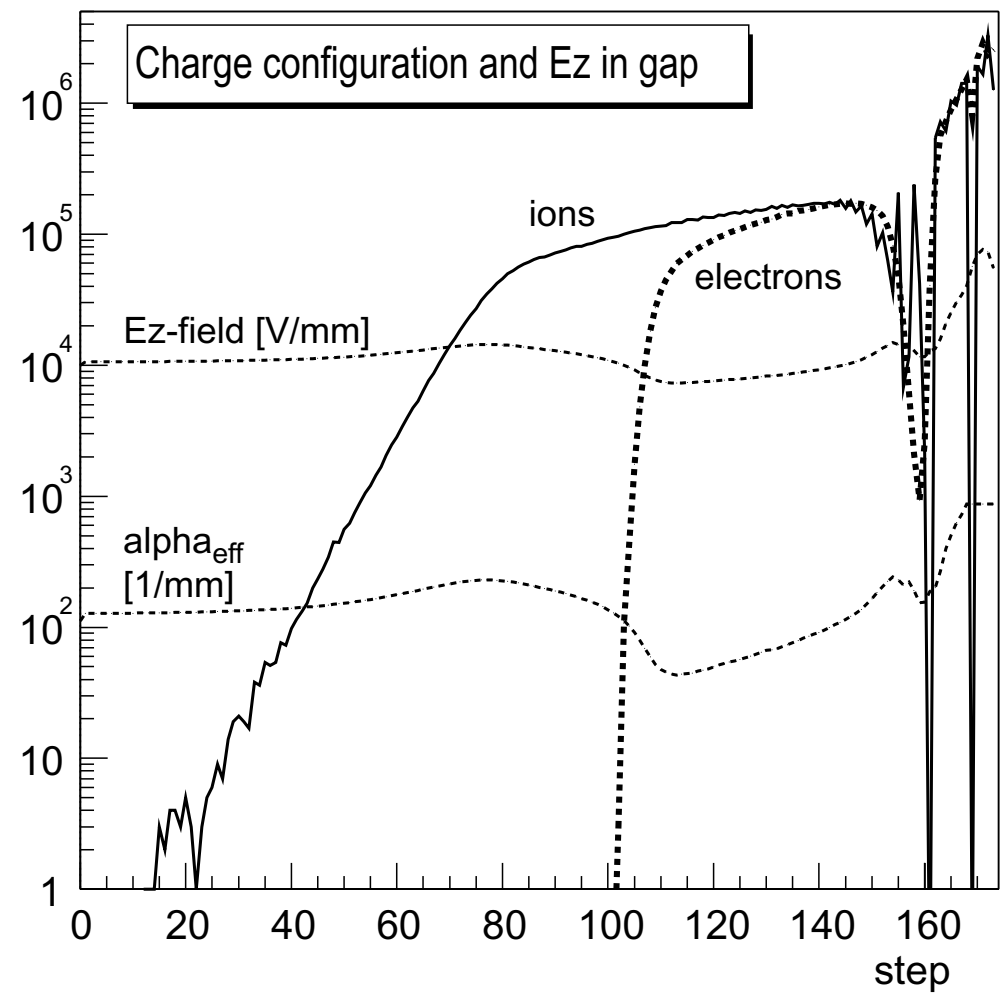

Fig. 2. Snapshot of an anode streamer. A $300 \mu \mathrm{m}$ gap is divided in 200 steps. The electric field and the effective Townsend coefficient $\alpha-\eta$ increase dramatically towards the tip of the avalanche. Each avalanche can only stay in saturated mode for a certain time. After this time the field will explode here. 

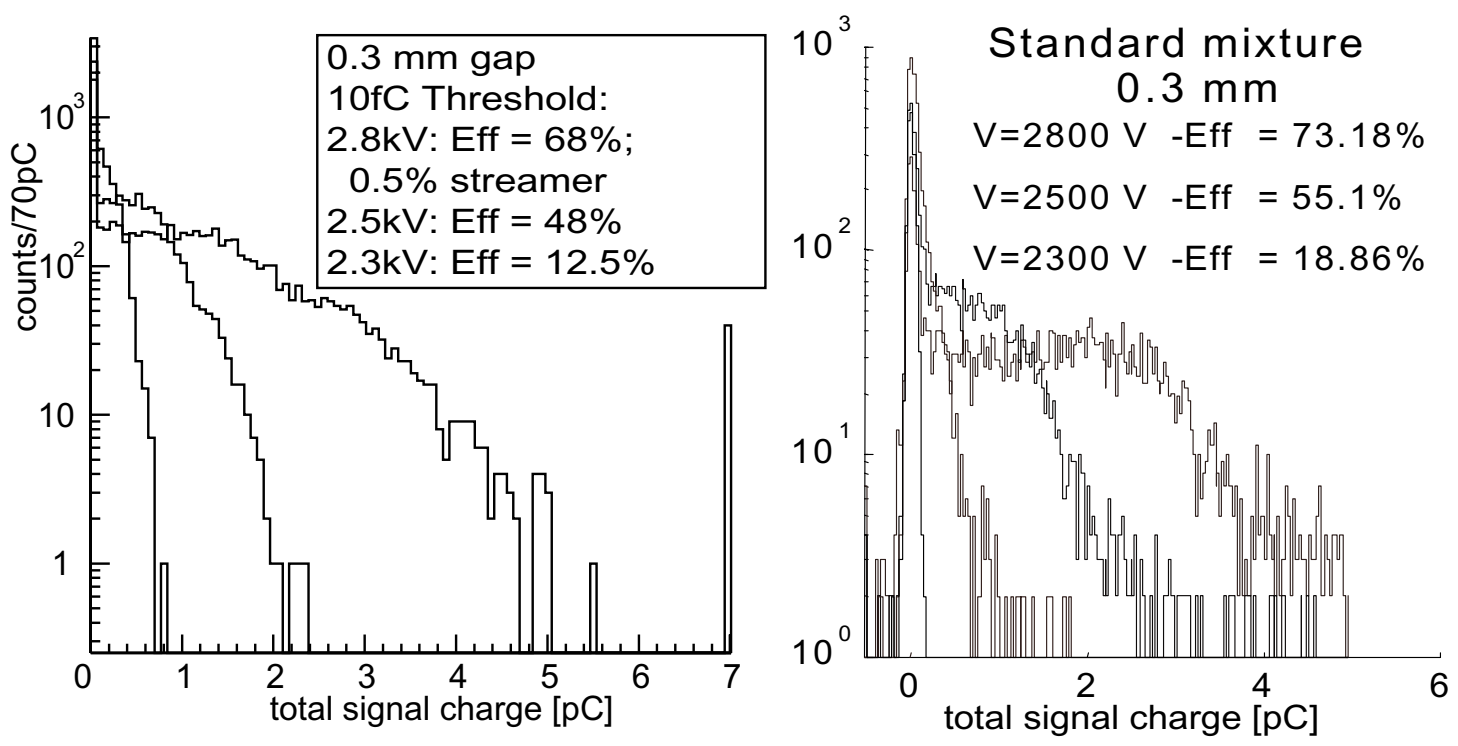

Fig. 3. Comparison of simulated and measured charge spectra for $300 \mu \mathrm{m}$ gap timing RPCs. The left image shows simulated spectra. Here the entries in the last bin are streamers. At $2.8 \mathrm{kV}$ we find $0.5 \%$ streamers. The right image was taken from [3]. Without space charge effect the average charge would be several orders of magnitude higher.

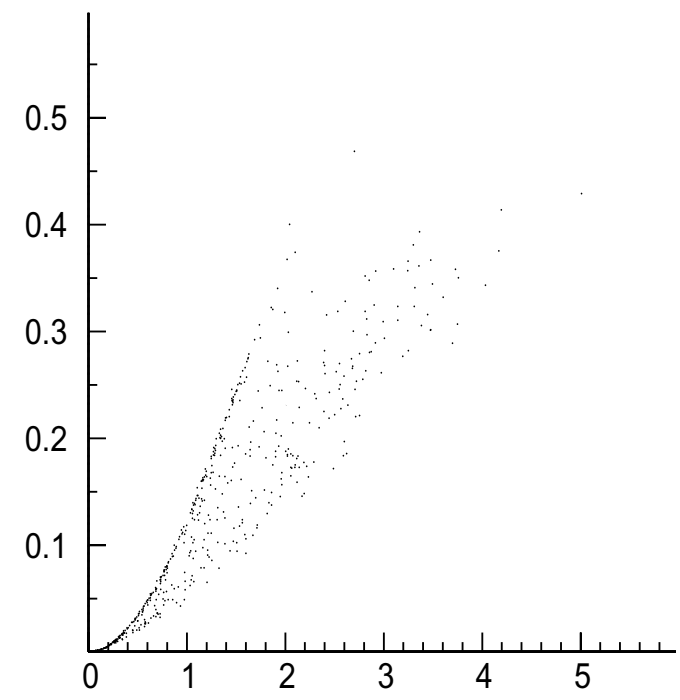




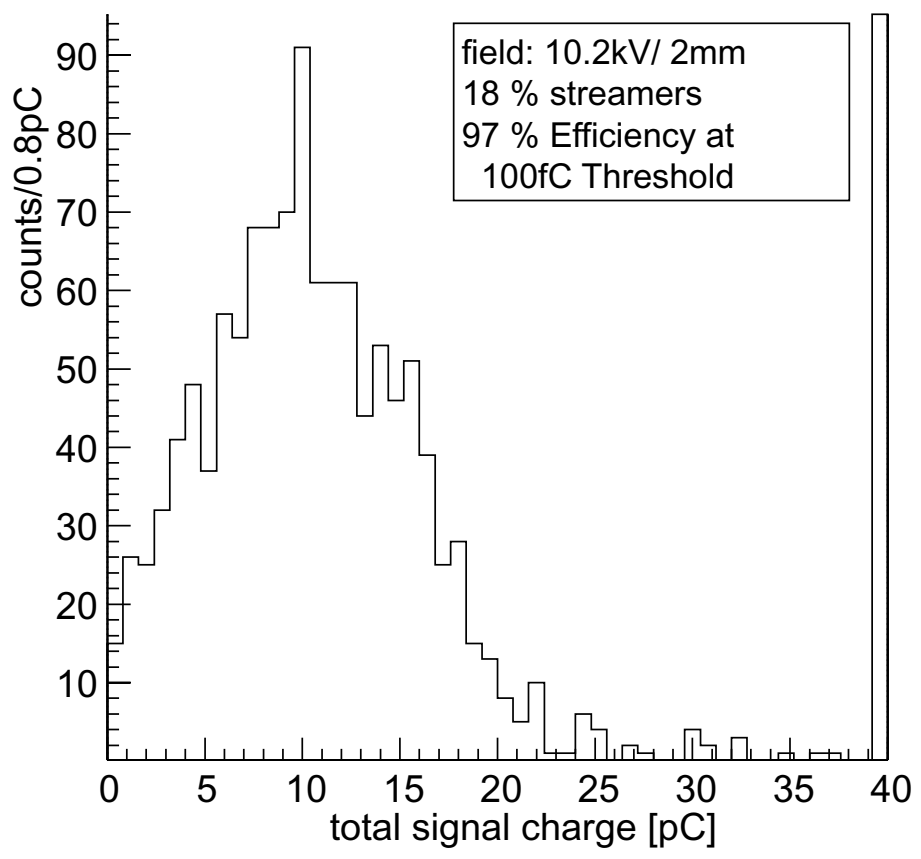

Fig. 5. Simulated total charge spectra for $2 \mathrm{~mm}$ gap trigger RPCs. The entries in the last bin are off-scale and correspond to anode streamers (18\%). The spectrum shows the characteristic peak also present in measurements.

[3] P. Fonte, V. Peskov, LIP-2000-04

[4] I. Smirnov, HEED, Program to compute energy loss of fast particles in gases, Version 1.01, CERN

[5] W. Riegler, C. Lippmann, Detailed Models for Timing and Crosstalk in Resistive Plate Chambers, RPC Conference Coimbra 2001

[6] S. Biagi, MAGBOLTZ, Program to compute gas transport parameters, Version 2.2, CERN

[7] S. Biagi, IMONTE, Program to compute gas transport parameters

[8] Th. Heubrandtner, B. Schnizer, C. Lippmann, W. Riegler, Static Electric Fields in an Infinite Plane Condensor with One or Three Homogeneous Layers, CERNOPEN 2001-074 